\title{
Research on Schedule Risk of Ship Maintenance Project Resource Allocation Based on System Dynamics
}

\author{
LIU Zhimeng ${ }^{1 \mathrm{st}, \mathrm{a}}$, YAO Lu ${ }^{2 \text { nd }}$, TANG Jiazheng ${ }^{3 \mathrm{rd} *}$ \\ ${ }^{1}$ Department of Management Engineering and Equipment Economics, Naval University of Engineering Wuhan, China \\ Liu Zhi-meng, Male, Graduate Student, Research option: Project Management \\ ${ }^{2}$ Department of Management Engineering and Equipment Economics, Naval University of Engineering Wuhan, China \\ ${ }^{3}$ School of Applied Science and Technology, Hainan University Wuhan, China
}

\begin{abstract}
Ship maintenance requires the use of a lot of resources, and the schedule risk caused by resource factors is an important reason for the long time and easy delay of maintenance projects. This paper adopts system dynamics theory to analyze the dynamic relationship between activity delay and resource allocation factors, and establishes a risk assessment model. Use anylogic software to simulate the implementation process of activities, and effectively simulate the mutual influence and change process of various risk factors, so as to achieve the purpose of accurately and effectively estimating the duration of project activities.
\end{abstract}

\section{INTRODUCTION}

In recent years, along with China's efforts to modernize its naval equipment and expansion of its missions, a large number of new ships are deployed for troops. This results in more frequent mobilization of ships and more complex environment for the use of vessels. These changes have put forward higher requirements for vessel maintenance and support capabilities ${ }^{[1]}$.

Due to its long maintenance period, naval vessel maintenance project is easy to be delayed. It not only leads to waste of military expenses, but also causes delay in the equipment maintenance plan to an extent that affects normal military deployment. ${ }^{[2]}$

The long period of maintenance period and project delay may be attributed to a number of factors. However, one of the key factors may be improper schedule arrangement due to conflict of resources. ${ }^{[3]}$

Due to its complicated systems and intensive technology, naval vessel projects may need to mobilize massive resources. Therefore, the key to main timely completion of the project with expected quality lies in how to properly allocate resources and work out a reasonable schedule.

In real maintenance, two challenges keep propping up. Firstly, schedule risk caused by improper allocation of resources is easy to be ignored, resulting in insufficient consideration of project period. So, in real practice, the project cannot be completed as per the schedule. Secondly, a loose schedule is provided with too much buffer time. Thus, the project may waste too much time due to insufficient use of time and resources. Therefore, the key to reasonable estimation of schedule lies in accurate assessment of schedule risks in resource allocation.

To address those challenges, this paper sorts out the general course of naval vessel resources schedule and summarizes the defects in conventional resource and period estimation. Based on the principle of system dynamics, upon studying causal relations between resource allocation risks and progress delay and analyzing the risk composition of resource allocation, this paper proposes a risk evaluation model consisting of initial risk, downstream risk, interaction risk, and risk factors. It uses anylogic to simulate the risk system. Various risks, the interactions between variables and the process of during the implementation period are simulated. The delay caused the risk system during the maintenance period is also quantified.

This paper aims to help project managers scientifically analyze schedule risk in resource allocation at the preparation stage, accurately estimate the time period for each activity, and work out a reasonable project schedule so as avoid project delay or perpetuation due to tight or loose arrangements.

\section{Project Resource allocation PRocess}

Resource management plays a pivot role in the whole project management. To assess the impact of resource allocation on project schedule, one needs to find out the process of resource allocation. 


\subsection{Signification of project resource allocation}

Resource allocation refers to the process of allocating the resource at the right time and right place. Proper resource allocation is the key to successful project implementation, as it is conducive to improvement of project indicators[4].

Naval vessel maintenance involves a variety of resources, which include human resources, material resources, financial resources and information resources[5]. Among them, maintenance personnel, facilities, equipment are man-made resources, which are not only the major types of resources in conflict but are the major factors to cause project delay. Therefore, this paper divides the maintenance resources into personnel, facilities and equipment to model the project schedule risk system.

\subsection{Resource planning process of maintenance project}

As shown in Figure 1, project resource allocation is closely knitted with project schedule management. Following this process, project activities can be identified with clear schedule and the required resource allocation plan.

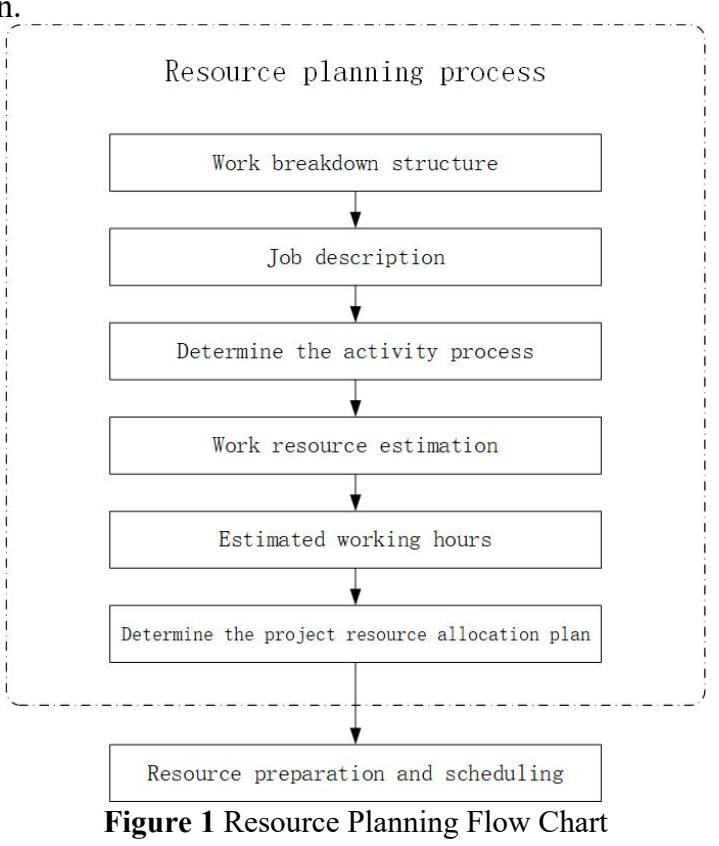

\subsection{Resource and schedule estimation}

Resource estimation and schedule estimation is to divide the work into independent and measurable breakdowns, and estimate the resources and period required for such breakdown.

2.3.1 Resource estimation can start from either the grassroots workers, who estimate the resources necessary for his share of work; or start from managers, who can directly optimize the resources plan based on bill of quantities, resources consumption standards, and historical records; In most cases, those two methods are combined together to finalize resource estimation.
2.3.2 For fixed activities, the completion time generally obeys a certain distribution [6]. According to the law of the distribution probability of the construction period, the estimated value of the construction period available for reference includes the maximum probability time, the average time, the optimistic time, and the pessimistic time. Wait. The common construction period value adopts empirical formula, or in critical chain technology, the average time Ta under 50\% completion rate is used as the estimated time of the active construction period [7].

\subsection{Necessity of research contents}

As the project resources need to be centralized in one space, collaborate together and sometimes are interchangeable [8], project resource allocation is not fixed but free in choice within certain scope. In different resource allocation plans, resource quantity, efficiency and progress risk may vary. All those differences caused by such discrepancy are easy to be ignored in the conventional resource allocation and estimation process, which in turn will lead to inaccurate estimation of maintenance period. Therefore, this paper specifically studies the resource allocation factors and explores the method of accurate estimation of activity duration. It is not only helpful for progress control, as it provides accurate timeline for resource scheduling and use, but also conducive to the smooth convergence of resources and activities. Therefore, it can greatly reduce the project period and avoid project delays.

\section{SYSTEM RISK ANALYSIS AND MODELING.}

In risk management, qualitative and quantitative methods are generally adopted to identify, assess and control risks.[9] Compare with ambiguous and inaccurate conventional risk management methods, system dynamics is good to address complex issues, which not only reflects the dynamic process of risk factors during project implementation but quantifies the impact of risk factors in the project. [10]

\subsection{Identification of risks}

\subsubsection{Model boundary determination}

Project progress is affected by a number of risk factors. This paper first identifies the progress risks from the perspective of project target. Then, it categorizes risk factors based on the types of resources and summarizes the structure of risk factors as shown in Figure2. 


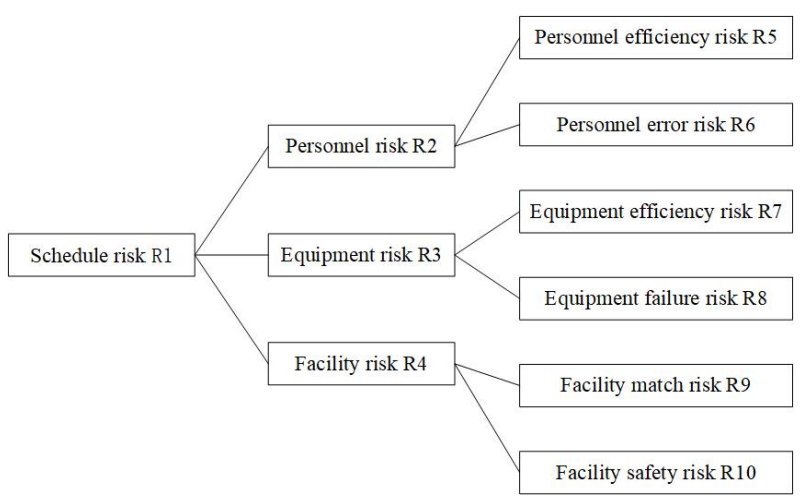

Figure 2 Structure Graph of Risk Factors for Vessel Maintenance Project

\subsubsection{Identification of causal relationship}

Causal loop diagram is established according to system dynamics model, shown in Figure 3.

This figure well reflects the structural relations between various risk factors and impact variables in the system. In the system, there are total 13 loops for progress risks, including 9 positive feedback loops and 4 negative feedback loops. It briefly implies that progress risks will magnify due to complex working of risk system.

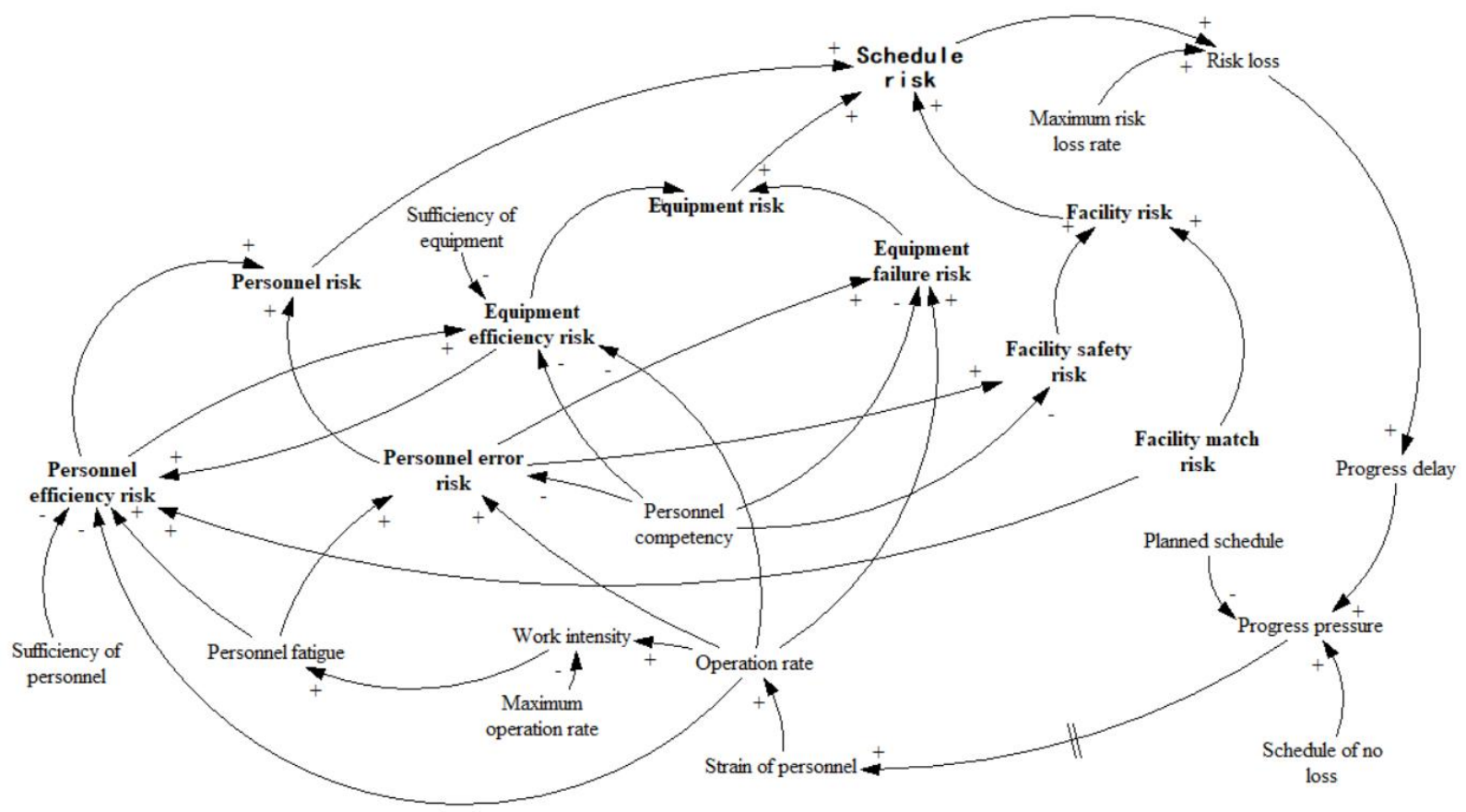

Figure 3 Causal Graph of Risk Factors for Vessel Maintenance Project

c. Interactive risks: risk variables due to interaction of

\subsection{Risk assessment and development of risk assessment model}

\subsubsection{Risk analysis}

Assessment of progress risk is based on the extent of impact on project schedule, including loss of time due to probability of risk events. This paper uses figure $0-1$ to assess the extent of risks and reflect the impact of risk factors on extension of schedule.

In the analysis system, the causes of risks are summarized into four aspects:

a. External risks: or initial risks, which is caused by factors outside the system. For instance, in assessment of risks, external factors such as political, financial and environmental factors should also be considered in addition to risks in personnel, facilities and equipment.

b. Downstream risks: Lower level of risk according to system categorization. For instance, progress risks consist of personnel risks, equipment risks and facilities risks. various risks in the system. For instance, personnel mistakes may reinforce equipment safety risks.

d. Risk factors: Variable factors that may affect the extent of risks. For instance, personnel mistakes risks may include personnel fatigue, personnel competency and work efficiency.

\subsubsection{Risk assessment model}

Risk assessment model is developed according to analysis of risk composition:

$R_{T}=R_{1} * R_{m}$

$R_{i}=R_{i}^{0}+R_{i}^{D}+R_{i}^{M}+R_{i}^{F}$

$R_{i}^{D}=\sum_{k \in D_{R i}} \alpha_{k} R_{k}$

$R_{i}^{M}=\sum_{j \in M_{R i}} \beta_{j i} R_{j}$

$\mathrm{R}_{\mathrm{i}}^{\mathrm{F}}=\mathrm{f}(\mathrm{A})$ 
Where, $R_{T}$ is risk loss, $R_{m}$ is progress loss rate corresponding to the maximum schedule risks. $R_{i}$ is the impact of risk i, $R_{1}$ is the system progress risk. $R_{i}^{0}, R_{i}^{D}$, $R_{i}^{M}, R_{i}^{F}$ respectively means the external risks, downstream risks, interactive risks and factor risks of risk i. $D_{R i}$ is the congregate of downstream risks for risk i; $M_{R i}$ is congregates of interactive risks for risk $\mathrm{i} ; R_{k}$ is the downstream risk of risk $\mathrm{i} ; R_{j}$ is the interactive risk of risk $\mathrm{i} ; \alpha_{k}$ is weight coefficient of downstream risk $\mathrm{k}$; $\beta_{j i}$ is interaction coefficient of interactive risk $\mathrm{j} . A$ is impact factors congregates in the system, $f(A)$ is the risk value generated by impact factors congregates.

\subsubsection{Establish System Flow Chart}

According to the causality diagram of risk system, this paper uses SD module of anylogic software to establish the system flow chart, as shown in Figure 4.

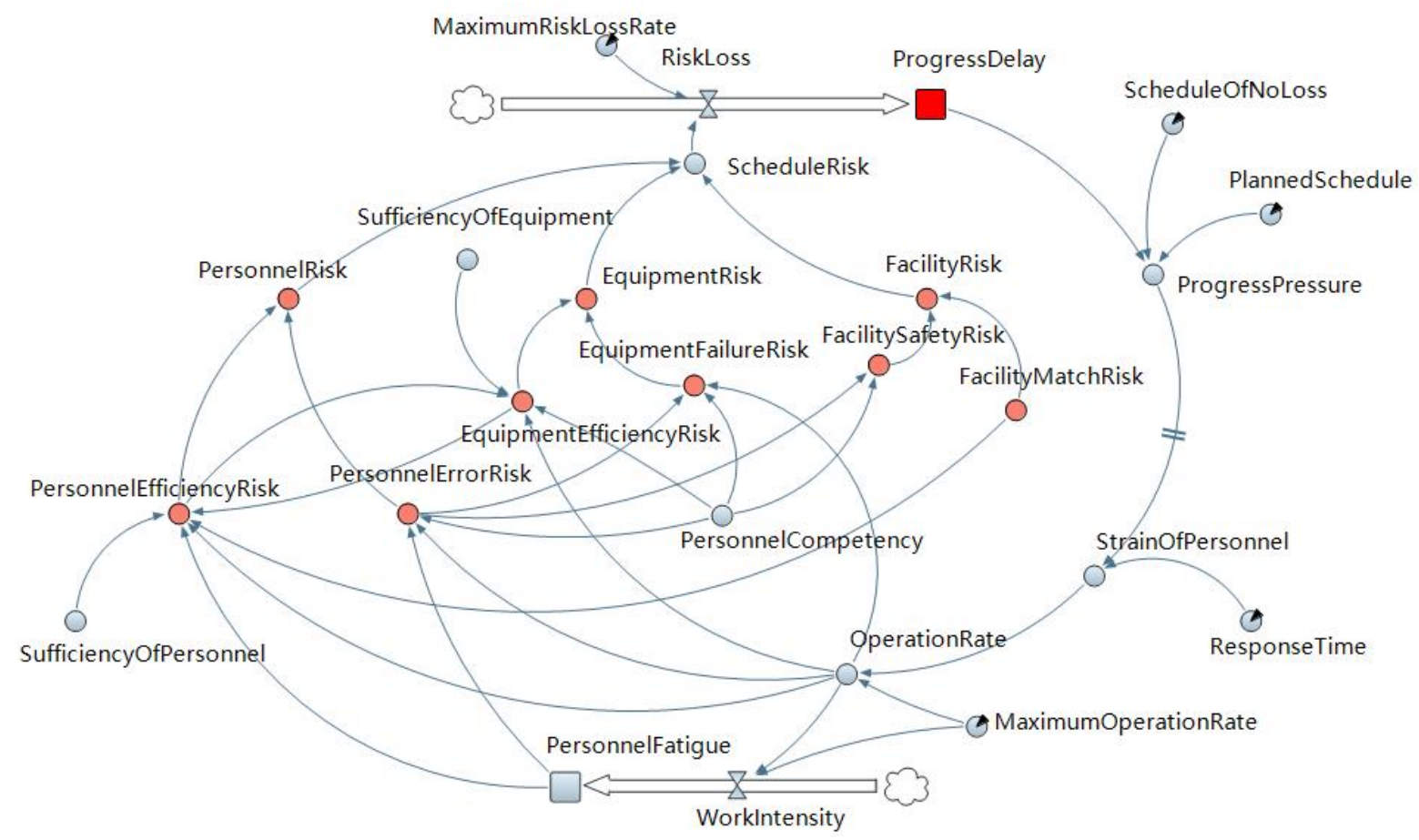

Figure 4 Flowchart of Progress Risks for Vessel Maintenance Project

\section{SimULATION AND RESULt ANALYSIS}

The maintenance certain patrol ship includes docking works, power works, electrical works, piping works, home decoration works, painting works, which can be further divided into 32 independent work breakdowns based on WPS. The section of main equipment inspection and maintenance in the power works has been allocated with the most optimal resource. In addition, no risk events will happen to affect its progress, its schedule of no loss will be 10 work hours. If general progress risks are considered, additional five hours will be considered for the planned schedule, which should be 15 work hours.

However, due to conflict of resources in the maintenance factory compounded with the requirement on optimization of resource allocation from various project activities, the resources allocated to main equipment inspection and maintenance cannot reach the best standards, so it is likely to result in progress risks. To accurately estimate the duration for this sectional works, this papers takes main equipment inspection and maintenance section as an example to model by adopting the method as stated.

\subsection{Parameter design and variables equation}

\subsubsection{Parameter design}

The assignment of risk impact and factor variables in the system shall follow certain criteria. In the risk assessment model proposed by this paper, the assignment values include initial risk value, risk weight coefficient, risk interaction coefficient and partial impact factors, which are set $0 \sim 1$ to keep consistency in the results. Based on the field investigation and relevant information, in combination with the scoring method proposed by experts, the assignment of project parameters is provided as Table 1:

Table 1 Major parameter assignment

\begin{tabular}{c|cc}
\hline & Type of parameters & Assignment \\
\hline \multirow{2}{*}{ Initial risk } & Schedule risk $\mathrm{R}_{1}{ }^{0}$ & 0.1 \\
& Personnel risk $\mathrm{R}_{2}{ }^{0}$ & 0.05 \\
\hline
\end{tabular}




\begin{tabular}{|c|c|c|}
\hline & $\begin{array}{c}\text { Equipment risk } \mathrm{R}_{3}{ }^{0} \\
\text { Facility riskR }{ }_{4}{ }^{0} \\
\text { Personnel efficiency risk } \mathrm{R}_{5}{ }^{0} \\
\text { Personnel error risk } \mathrm{R}_{6}{ }^{0} \\
\text { Equipment efficiency risk } \mathrm{R}_{7}{ }^{0} \\
\text { Equipment failure risk } \mathrm{R}_{8}{ }^{0} \\
\text { Facility match risk } \mathrm{R}_{9}{ }^{0} \\
\text { Facility safety risk } \mathrm{R}_{10}{ }^{0}\end{array}$ & $\begin{array}{c}0.05 \\
0.04 \\
0.1 \\
0.05 \\
0.15 \\
0.05 \\
0.4 \\
0.02 \\
\end{array}$ \\
\hline \multirow{9}{*}{ Weight coefficient } & $\alpha_{2}$ & 0.4 \\
\hline & $\alpha_{3}$ & 0.4 \\
\hline & $\alpha_{4}$ & 0.2 \\
\hline & $\alpha_{5}$ & 0.5 \\
\hline & $\alpha_{6}$ & 0.5 \\
\hline & $\alpha_{7}$ & 0.4 \\
\hline & $\alpha_{8}$ & 0.6 \\
\hline & $\alpha_{9}$ & 0.3 \\
\hline & $\alpha_{10}$ & 0.7 \\
\hline \multirow{5}{*}{$\begin{array}{l}\text { Interaction } \\
\text { coefficient }\end{array}$} & $\beta_{7,5}$ & 0.3 \\
\hline & $\beta 9,5$ & 0.4 \\
\hline & $\beta_{5,7}$ & 0.3 \\
\hline & $\beta_{6,8}$ & 0.6 \\
\hline & $\beta_{6,10}$ & 0.7 \\
\hline \multirow{3}{*}{ Impact factors } & Sufficiency of personnel & 0.9 \\
\hline & Sufficiency of equipment & 0.7 \\
\hline & Personnel competency & 0.8 \\
\hline \multirow{2}{*}{ Other parameters } & Maximum risk loss rate & 0.8 \\
\hline & Response time $(\mathrm{T})$ & 0.5 \\
\hline
\end{tabular}

\subsubsection{Establishment of system equation}

Major system equations are established according to the risk model and the law of risk impact factors proposed in this paper:

a. Progress delay $=\mathrm{INTEG}$ (risk loss, no time loss)

b. Risk loss $\mathrm{RT}=$ Schedule risk $\mathrm{R} 1 *$ maximum risk loss rate $\mathrm{Rm}$

c. Schedule risk R1=0.1+0.4*R2+0.4*R3+0.2*R4

d. $\mathrm{R} 2=0.05+0.5 * \mathrm{R} 5+0.5 * \mathrm{R} 6$

e. $\mathrm{R} 3=0.05+0.4 * \mathrm{R} 7+0.6 * \mathrm{R} 8$

f. $\mathrm{R} 4=0.04+0.3 * \mathrm{R} 9+0.7 * \mathrm{R} 10$

g. $\mathrm{R} 5=0.1+0.3 *$ Equipment efficiency

risk $+0.4 *$ Equipment match risk $+3 *$ Math.pow (1-

Sufficiency of personnel,2) $+0.1 *$ Personnel fatigue-

$0.8 *($ Operation rate- 1$)$

h. R6 $=0.05+3 *$ Math.pow(1-Personnel competency,2) $+0.1 *$ Personnel fatigue $+0.5 *$ Math.pow (Operation rate$1,2)$

i.R7 $=0.15+0.3 *$ Personnel efficiency risk $+0.4 *(1-$ Personnel competency) $+3 *$ Math.pow(1-Sufficiency of equipment, 2$)-1.2 *$ (Operation rate- 1 )

j. $\mathrm{R} 8=0.05+0.6 *$ Personnel error risk $+0.4 *(1-$ Personnel competency) $+2 *$ Math.pow(Operation rate$1,2)$

k. R9=0.4

$1 . \mathrm{R} 10=0.02+0.7 *$ Personnel error $\operatorname{risk}+0.3 *(1-$ Personnel competency)

m. Progress pressure $=\min ($ Progress delay $/($ Planned schedule-Schedule of no loss ),1)

n. Strain of personnel=delay (Progress pressure, Response time)

o. Operation rate $=1+$ Strain of personnel*(Maximum operation rate-1) p. Work intensity $=$ (operation rate $/$ maximum operating rate)*0.2

q. Personnel fatigue $=$ INTEG (Work intensity, time without loss)

\subsection{Simulation operation and result analysis}

\subsubsection{Simulation operation}

Set the simulation time to be the schedule of no loss, i.e. 10 hours. During the simulation period, the trend of change for progress risks caused by schedule risk and resource allocation factors is shown in Figure 5 and Figure 6. See Figure 7 to acquire the extent of impact of progress risks on project schedule.

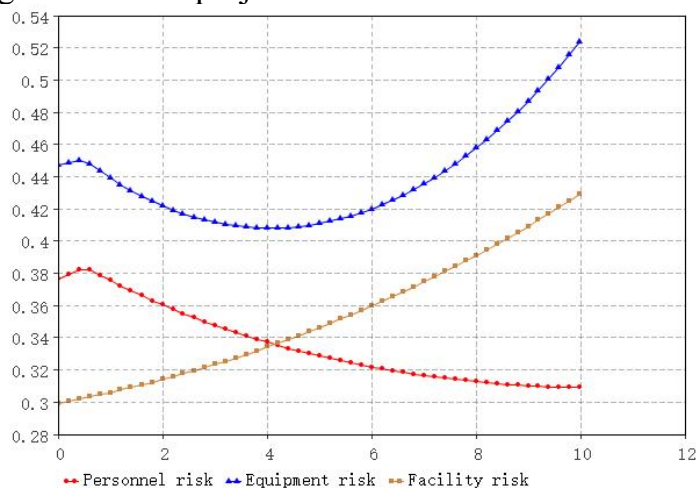

Figure 5 Time Line Chart of Resource Schedule Risk 


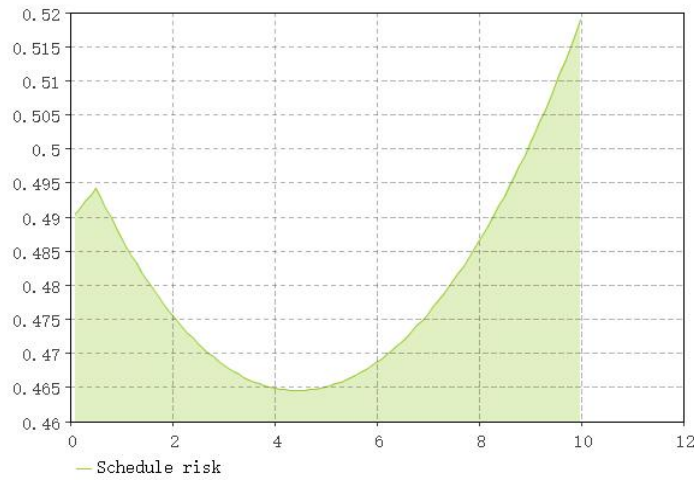

Figure 6 Time Line Chart of Schedule Risk

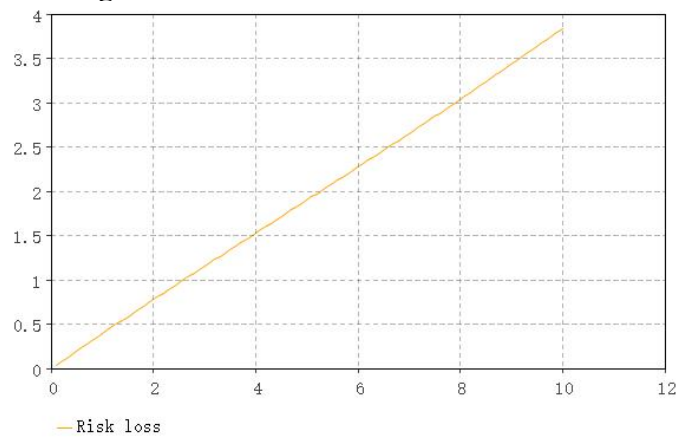

Figure 7 Time Line Chart of Project Delay

\subsubsection{Result analysis}

Simulation results: A, the vessel maintenance project can be completed in the planned period of 15 work hours under current allocation of resources and environment. B, by simulation, the delay is 3.87 hours, with 1.13 hours less than conventional buffer time. So there is waste of time in the planned schedule. $\mathrm{C}$, the planned schedule is adjusted to 14 hours. By further simulation, the acquired delay is 3.94 hours, less than or close to 4 hours of buffer time. This shows that it is more reasonable to set 14 hours as the planned maintenance period.

\section{ConCLusion}

By exploring the law of the impact of resource allocation on vessel maintenance project, this paper puts forward a schedule risk evaluation model made of four parts. It adopts system dynamics theory to analyze the causal relationship between delay and resource allocation factors and establishes a risk system. The impact of resource allocation factors on the project schedule are quantified to improve accuracy in schedule estimation, thus reducing the project period and avoiding delay. The model in this paper can serve as a basis or project progress management. However, the relations between the risk variables need to further explored so as to improve the accuracy in real schedule estimation.

\section{REFERENCES}

1. Li ZhongmengResearch on the Development of warship equipment. Journal of Ordnance Equipment Engineering,2019,40(12):131-135.

2. Yan Gongda,Dong Peng,Yu Peng,Shao Shuai.The application of HHM in the risk identification of ship maintenance schedule.Ship Electronic Engineering,2019,39(02):132-136.

3. K.Salimifard, G. Jamali, et al.Resolving Resource Conflicts in a Ship Repair Project .International Journal of modeling and Optimization,2012,2(5):618-620.

4. Vinayak C. Sawant. Genetic Algorithm for Resource Constrained Project . International Journal of Science and Research,2016,5(6):139-146.

5. Yang Chao, Hou Xingming, Liao Xinghe, Qin Haifeng, Xu Qing. Literature analysis and content review of equipment maintenance support resource allocation.Chinese Journal of Ordnance Equipment Engineering,2019,40(07):170-175.

6. Liu Zhiqiang. Research on the progress control of ZB ship equipment system mid-repair project. South China University of Technology, 2011.

7. Xiao Qiming. Research on optimization of engineering project schedule based on improved critical chain. East China Jiaotong University, 2018.

8. Xiong Ying. Theory and Method of Resource Optimal Allocation for Construction Project. Beijing Jiaotong University, 2008.

9. Lian Qichao. Risk analysis and countermeasure research of construction project based on fuzzy analytic hierarchy process. Xi'an Polytechnic University, 2019.

10. Li Cunbin,Lu Gongshu.System dynamics model of project risk element transmission.System Engineering Theory and Practice,2012,32(12):27312739. 